\title{
Improving customs' border control by creating a reference database of cargo inspection X-ray images
}

Selina Kolokytha ${ }^{* 1}$, Alexander Flisch ${ }^{1}$, Thomas Lüthi ${ }^{1}$, Mathieu Plamondon ${ }^{1}$, Adrian Schwaninger ${ }^{2}, 3$, Wicher Vasser ${ }^{3}$, Diana Hardmeier ${ }^{3}$, Marius Costin ${ }^{4}$, Caroline Vienne ${ }^{4}$, Frank Sukowski ${ }^{5}$, Ulf Hassler ${ }^{5}$, Irène Dorion ${ }^{6}$, Najib Gadi ${ }^{6}$, Serge Maitrejean ${ }^{6}$, Abraham Marciano ${ }^{6}$, Andrea Canonica ${ }^{7}$, Eric Rochat ${ }^{7}$, Ger Koomen $^{8}$, Micha Slegt ${ }^{8}$

${ }^{1}$ Empa - Swiss Federal Laboratories for Materials Science and Technology, Center for X-ray Analytics, 8600 Dübendorf, Switzerland

${ }^{2}$ University of Applied Sciences and Arts Northwestern Switzerland, School of Applied Psychology,4600 Olten, Switzerland

${ }^{3}$ Center for Adaptive Security Research and Applications (CASRA), 8050 Zürich, Switzerland

${ }^{4}$ CEA, LIST, Department of Imaging \& Simulation for Non-Destructive Testing, 91191 Gif-sur-Yvette, France

${ }^{5}$ Fraunhofer Institute for Integrated Circuits IIS, Development Center for X-ray Technology (EZRT), 90768 Fürth, Germany

${ }^{6}$ Smiths Heimann S.A.S. (SH), 94405 Vitry sur Seine, France

${ }^{7}$ Swiss Federal Customs Administration (FCA), 3003 Bern, Switzerland

${ }^{8}$ Dutch Customs Laboratory (DTCA), 6401 DN Heerlen, The Netherlands

A R T I C L E IN F O

Article history:

Received: 16 February, 2017

Accepted: 22 March, 2017

Online: 06 April, 2017

Keywords :

$X$-Ray

Inspection

Cargo

Database

Imaging

Security

Training

\begin{abstract}
A B S T R A C T
Countries' effective and efficient border security is becoming increasingly important in today's global world of economy and trade. To ensure these, customs organisations are responsible for the prevention of illicit goods' transportation across borders, particularly upon entry. The predicament of the customs administrations will soon be aggravated by both the increase of global trade and the trend towards $100 \%$ screening. It is therefore a goal to advance inspection methods to enable successful cargo handling, a scope towards which this research was aimed at. This work was carried out as part of the project ACXIS "Automated Comparison of X-ray Images for cargo Scanning" a European research project within the seventh framework programme answering the call SEC-2012.3.4-1: " Research on Automated Comparison of X-ray Images for cargo Scanning », to improve the process with the largest impact to trade flow: the procedures of freight X-ray scanning. As such, this project was focused on to implementing a manufacturer independent reference database for X-ray images of illicit and non-illicit cargo, developing procedures and algorithms in order to uniform $X$-ray images of different cargo scanners, and developing a training simulator for inspection officers and a toolbox enclosing several assisted and automated identification techniques for potentially illicit cargo.
\end{abstract}




\section{Introduction}

Within the grand scheme of security, border control, and specifically the screening of border traffic cargo, has seen increasing focus in recent years. Given today's world of trade and the threats that exist to our societies, effective and efficient border controls are increasingly essential. The fiscal integrity and security of the movements of goods across these borders are the responsibility of customs administrations. Accordingly, they are also expected to have a positive impact on the economic competitiveness of their respective nation.

Indeed, customs administrations aim at identifying cargo that is inconsistent with its declaration, contain illicit goods, or that pose threats to society. Smuggling of counterfeited and pirated goods cause harm to a nation's wellbeing due to missed taxes that could otherwise be put to service for society. The Organization for Economic Co-operation and Development (OECD) estimates that the global international trade in counterfeits and pirated goods in 2007 may amount to USD 250 billion, which would equate to $1.95 \%$ of global trade [1]. Cigarettes lead the chart in the European Union with around $35 \%$ among all smuggled goods [2]. The increasing number of cargo transported through borders requires innovative inspection procedures. In this direction, automatic detection in cargo screening constitutes an important role.

Within the project ACXIS, cargo screening developments are made in supporting the inspection officers' work of illicit cargo detection. More specifically, ACXIS develops automated target recognition (ATR) functions to analyse X-ray images of cargo screened at border crossing points (land, air or sea). These functions can continuously self-improve through a central reference data base. This database contains X-ray images of illicit and non-illicit cargo, and it is designed to be manufacturer independent. All these database images, particularly the ones containing threats, constitute a significant tool for the training of screening officers and also for machine learning methods in automated detection algorithms [3,4].

\section{Hardware Set-Up and Optimisation}

In order to image illicit cargo mock-ups, laboratory X-ray scanners were used at Empa - the Swiss Federal Laboratories for Materials Science and Technology, at CEA - the French Alternative Energies and Atomic Energy Commission, and at EZRT - the German Development Center for X-ray Technology [5]. The Dutch and Swiss customs administrations used their own commercial cargo scanners, such as Smiths Heimann, Rapiscan and Nuctech systems.

The X-ray source used for the data acquisition at Empa was a portable dual energy 4 and $6 \mathrm{MeV}$ linear accelerator; with a focal spot size of $2 \mathrm{~mm}$ (Fig. 1). Certain modifications were made on the existing high energy CT at Empa, in order to optimise the system for this project [6-8]. First, rails were placed on the linear accelerator and the detector towers to allow changing the sourceto-detector distance up to $5.4 \mathrm{~m}$. Then, the CT detector were replaced by a newly designed line detector consisting of 20 modules with 32 channels each, made of $30 \mathrm{~mm}$ deep CdWO4 scintillators with a pitch of $4.6 \mathrm{~mm}$ (X-Scan LCS 4.6, from Detection Technology). A 3D CAD of the new detector is shown in Fig. 2. Careful design and work was carried out in the detector assembly in reducing X-ray scattering. Additionally, new lead and tungsten source collimators were installed to shape the beam according to the detector geometry. An X-ray filter, specially designed, was fitted between the primary and secondary source collimators to flatten the intensity of the X-ray beam over the full detector width (approximately $3 \mathrm{~m}$ ).

The laboratory scanner built at CEA consists of a linear accelerator, a line detector and a translation table (Fig. 3). The scanning geometry was chosen to match the commercial scanners, namely a horizontal translation of the object, using a vertical detector line. The linear accelerator is a Varian Linatron M9A model, delivering 5, 6 and $9 \mathrm{MeV}$ with a maximal dose rate up to $30 \mathrm{~Gy} / \mathrm{min}$. The focal spot size is specified to less than $2 \mathrm{~mm}$ in diameter. The detector is made of $10 \mathrm{X}$-Scan LCS 4.6 modules, assembled on a straight line. A lead collimator is used in front of the active area and the backside is shielded by $5 \mathrm{~mm}$ of lead.

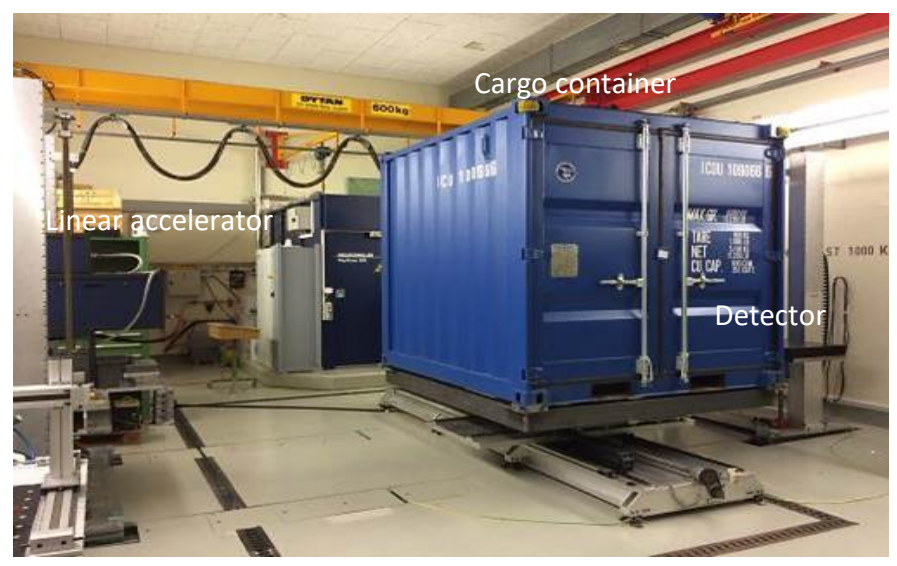

Figure 1 ACXIS laboratory set up of cargo container imaged by linear accelerator (left) and new detector (right; hidden behind container).

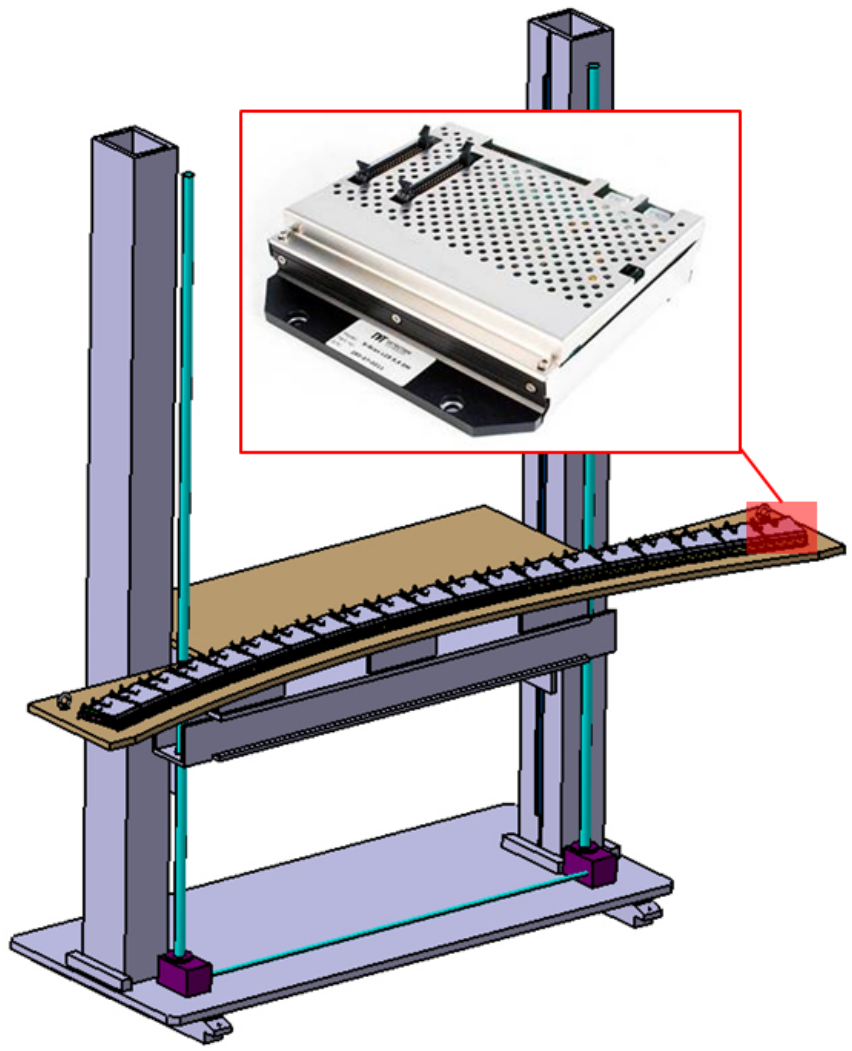

Figure $23 \mathrm{D}$ CAD of the new detector, shown without the casing, to allow visualisation of the individual detector modules, one of whose close up photo is shown. The CAD shows the detector fitted on adjustable towers. 


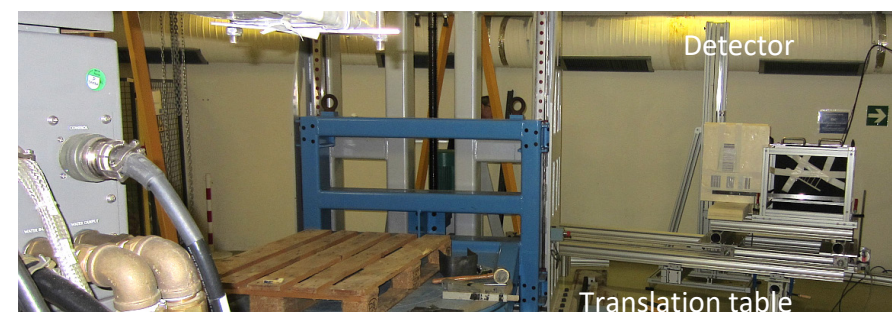

Figure 3 Laboratory setup at CEA.

The translation table has a second plate that can be tilt with $\pm 15^{\circ}$, as shown in Fig. 4. This option was used for mock-up objects for which different incident views were needed. Tilting the objects is equivalent to placing them at different heights in a full container, hence several combinations of various positions were operated more easily.

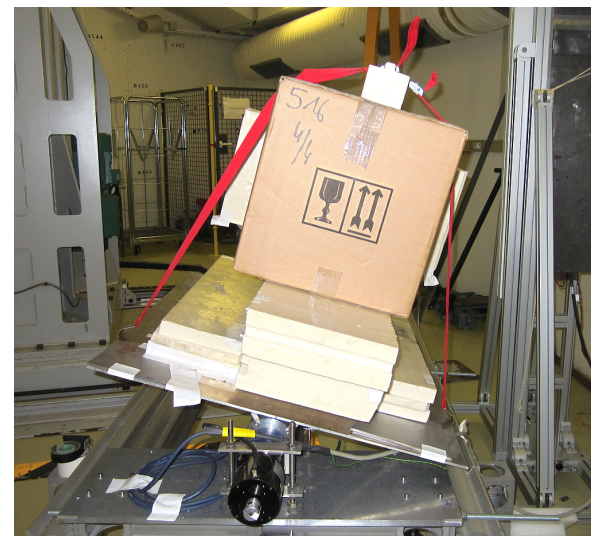

Figure 4 Translation table with tilt in the setup of CEA.

\section{Reference Database: An Integration}

One of ACXIS key goal is the creation of a reference database of realistic X-ray images of both non-illicit and illicit cargo. Such a database is essential for the development of training systems for customs officers and for providing assisted detection techniques of threats with the use of dedicated algorithms. To construct this database, we rely on historic images provided by customs. However, the number of threat images acquired through real detections remains limited and needs to be increased by additional threat images obtained through simulation. A very important aspect for this project is that all the pre-mentioned images must have a standard format so that images originating from different systems can be incorporated.

\subsection{Creating Artificial Images of Illicit Cargo}

To efficiently generate X-ray images for the database we can rely on two strategies. The first one consists in using simulation tools to generate artificial X-ray projections of illicit cargo. CEA develops the software CIVA, which integrates a module for modelling X-ray generation, interaction and detection, thus allowing the creation of realistic radiographic projections of any object that is put in the simulation scene. In this case, the shape of the object has to be described by a 3D CAD model and its material has to be specified. For multi-material objects however, each material component has to be segmented, which will result in highly complex objects in the case of a complete cargo. In practice, due to the complexity of container loadings, this approach has not been implemented. The second strategy consists in using real images of legal freights, which are widely available, and to combine these with one or several threat images. For this purpose a so-called "merging tool" has been developed and threat images have been either acquired or created by simulation.

Both approaches have advantages and disadvantages: for instance, cigarettes packs have a very specific texture that is difficult to model in the simulation software and real images have to be used in this case. On the other hand, some realistic 3D models of weapons have been collected and were used to easily generate multiple threat images with various orientations.

\subsection{Threat Images}

\subsubsection{Acquisition from Mock-Up Scans}

Based on risk analysis conducted by the customs administrations, threat objects and their locations in the container were identified. Following their recommendations, a set of mockups was created with real threat items and simulants, combined with common goods (Figure 5). These were scanned using laboratory systems, and some with a cargo scanner, chosen as reference.

For some illicit items the incidence of the X-ray paths through the object largely influences the produced image. Therefore mockup X-ray scans were performed with different object tilts so that the best projection could be selected depending on the vertical position of the object in the container.
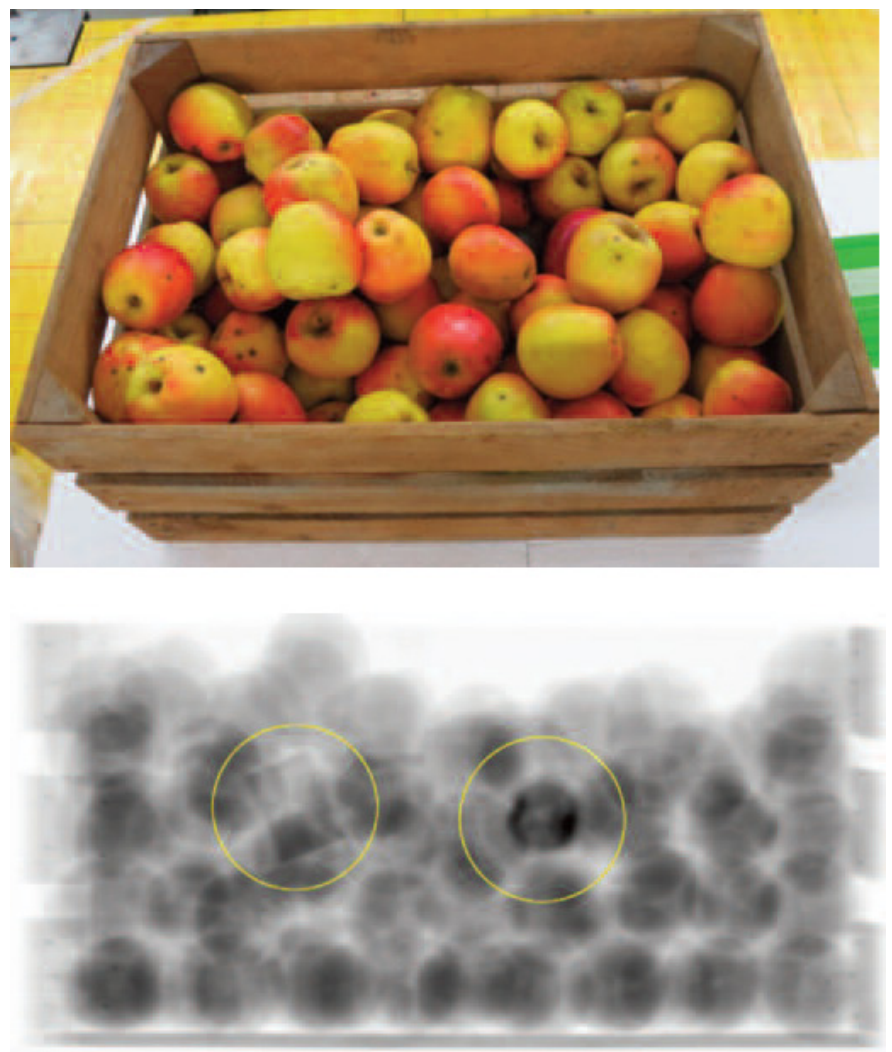

Figure 5 Photograph of a container of apples containing hidden Plastex (left) and hand grenade (right), above, and X-ray image of this threat container, below.

\subsubsection{Generation from Simulation Software}

The main objective of the mock-up scans was to enlarge the variety of threat images. For the same purpose, simulation tools 


\section{S. Kolokytha et al. / Advances in Science, Technology and Engineering Systems Journal Vol. 2, No. 3, 60-66 (2017)}

were also used in order to generate X-ray images from 3D models of various types of threat items (e.g. weapons).

The acquisition geometry, source spectrum and detector parameters of the high energy scanner were defined in the simulation software. 3D models of different weapons were then loaded into the software environment and multiple projections were created (Figure 6).
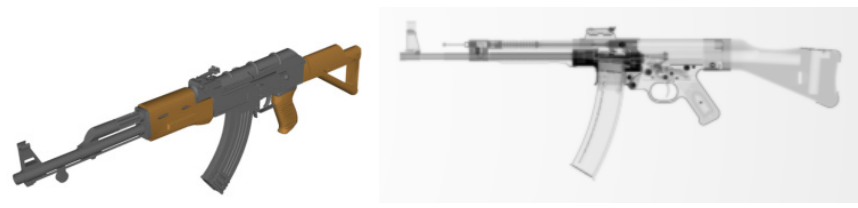

Figure 6 3D CAD model (left) and simulated X-ray projection (right) of a weapon.

The strength of the simulation approach is to create easily a large set of images of an object from different viewpoints. This variety of images of a same object is essential for training learning algorithms for detecting specific threats. Figure 7 displays simulated images of a small weapon, a larger weapon and a bottle that could represent a chemical product used for explosive devices for instance. Each threat has been rotated from side view to top view by steps of $30^{\circ}$.

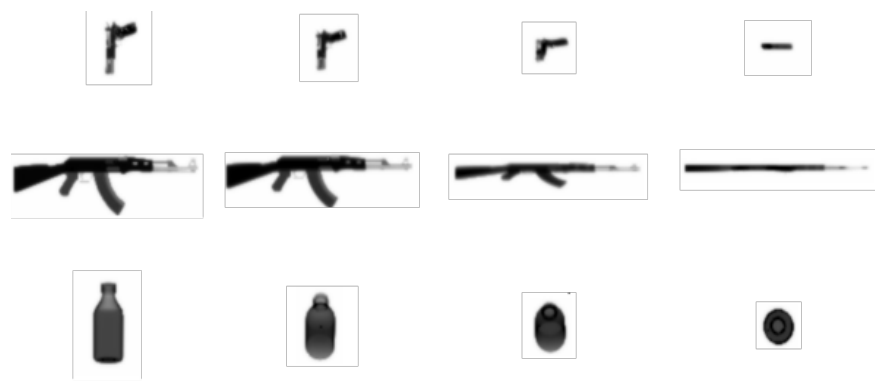

Figure 7 Simulated images of three different threats from multiple viewpoints.

\subsection{Merging Threat Images with Historic Ones}

The basic principle of X-ray imaging is the measurement of the $\mathrm{X}$-ray photons which are attenuated when traversing matter. This process is modelled by the Beer-Lambert law, which can be approximated as follows:

$$
I_{\text {det }}=I_{0} e^{-\sum_{i=1}^{N} \mu_{i} \cdot x_{i}}
$$

Where $I_{\text {det }}$ is the beam intensity at the detection point after traversing $\mathrm{N}$ different materials, $\mathrm{I}_{0}$ is the beam intensity at the initial point, $\mu_{i}$ and $x_{i}$ are the linear attenuation coefficient and the thickness of each material, respectively. According to (1), the beam intensity at the detector point corresponds to the combination of the attenuation due to each material successively penetrated by the X-ray beam. It can be decomposed as:

$$
I_{\text {det }}=\underbrace{I_{0} \cdot e^{-\mu_{1} \cdot x_{1}} \cdots e^{-\mu_{j} \cdot x_{j}}}_{I_{b g}} \cdot \underbrace{e^{-\mu_{j+1} \cdot x_{j+1}} \cdots e^{-\mu_{N} \cdot x_{N}}}_{e^{-\mu_{\text {th }} \cdot x_{\text {th }}}}
$$

Where $\mathrm{I}_{\mathrm{bg}}$ is the beam intensity attenuated by the background (non-illicit cargo without threat constituted of $\mathrm{j}$ materials) and the parameters $\mu_{\text {th }}$ and $x_{\text {th }}$ represent the characteristics of the threat element (constituted of $\mathrm{N}-\mathrm{j}$ materials).

Then, by injecting in (2) the additional measure of the incident beam intensity attenuated by the threat $\mathrm{I}_{\text {th }}$ it comes:

$$
\mathrm{I}_{\mathrm{det}}=\mathrm{I}_{\mathrm{bg}} \cdot \frac{\mathrm{I}_{\mathrm{th}}}{\mathrm{I}_{0}}
$$

with:

$$
\mathrm{I}_{\mathrm{th}}=\mathrm{I}_{0} \cdot \mathrm{e}^{-\mu_{\mathrm{th}} \cdot \mathrm{x}_{\mathrm{th}}}
$$

According to (3), we can combine pixel to pixel the attenuation of the threat (threat image acquired as described in earlier subsection) to the grey value of the non-illicit cargo image to create an image of illicit cargo (Figure 8).

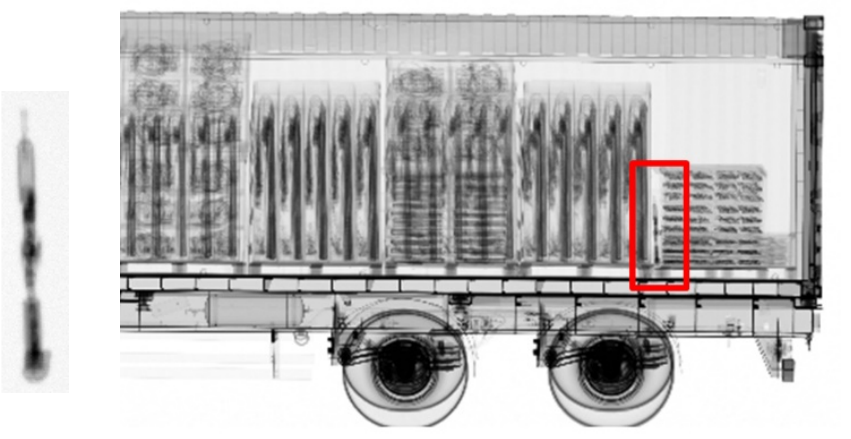

Figure 8 Simulated image of a weapon (left) inserted into a non-illicit cargo to create an artificial illicit cargo image (right).

The implemented tool allows the insertion of a same threat image multiple times at different positions in the background image and with different orientations. The major difficulty when performing the merging is in the selection of suitable and realistic spots for placing the considered threat.

A first set of illicit cargo images created artificially has been submitted to customs officers, who have assessed and validated the quality of the merging process.

\subsection{Creating a Unified X-ray Image Standard}

Upon visual inspection of X-ray images, the human brain can easily adapt to different systems, regardless the different parameter variations such as geometric deformations, textures, and levels of contrast or noise. This is not equally the case for automatic algorithms.

Given the large variety of X-ray scanners installed at border checkpoints, it is important that the acquired images are similar in terms of the permissioned parameters. As such, standardising these images enables an increase in the automatic detection performances. Therefore, all X-ray data can be converted into a standardised format and stored in a database, along with the results of the ATRs and useful annotations (Figure 9 centre). This standardisation enables the comparison between scans originating from different X-ray imaging systems. The database consists of set of approximately 30.000 scans as reference material, and new scans can be integrated continuously. 

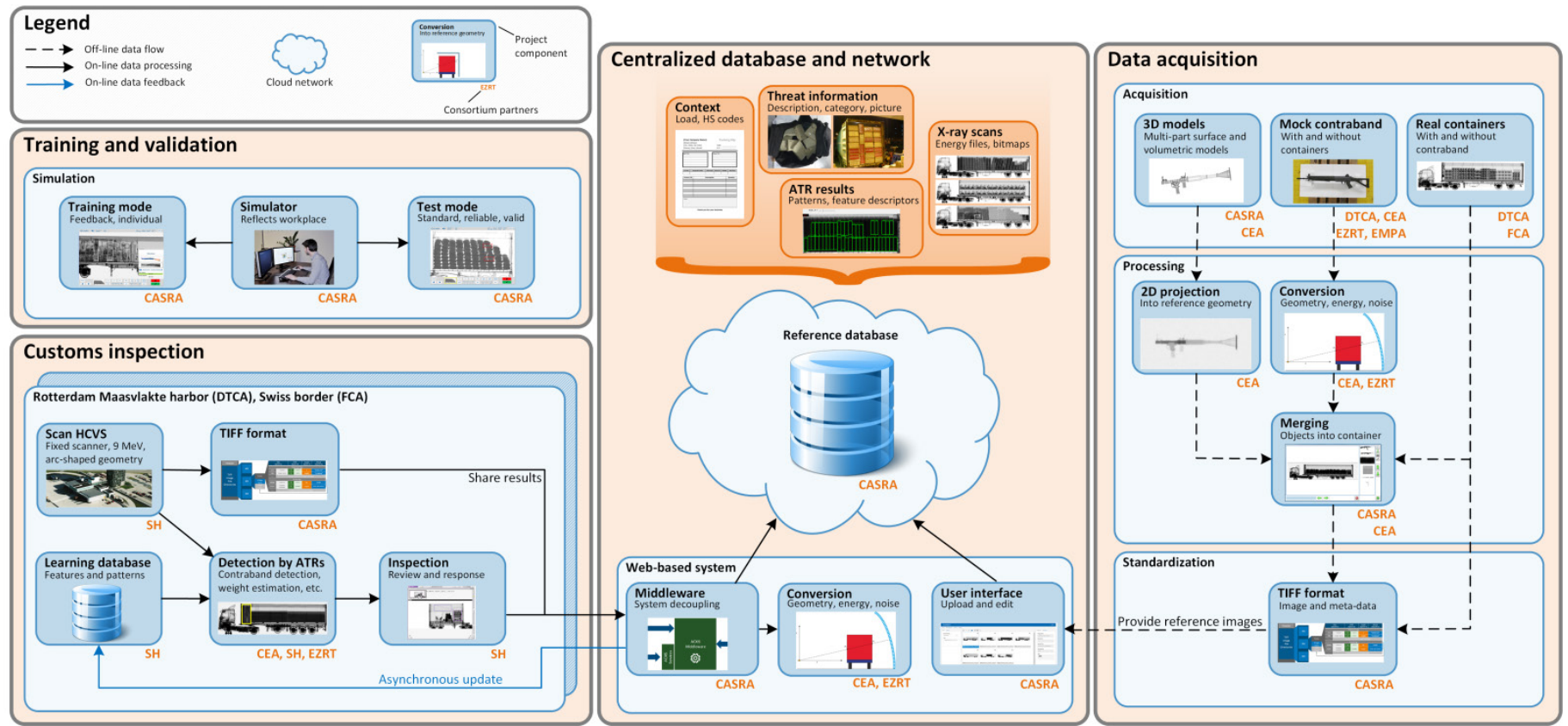

Figure 9 X-ray inspection procedure for border controls, with automated target recognition functions based on a centralised reference database.

Similarly, scanners with very different energy spectra such as mobile systems equipped with $2.5 \mathrm{MeV}$ sources and stationary ones using $9 \mathrm{MeV}$ accelerators produce very different images in terms of contrast and image quality [9]. Based on this analysis, it is not relevant to convert all scanner images to a unique reference configuration but for creating a homogeneous database, we propose to standardise images acquired with systems of energy in the range $4 \mathrm{MeV}$ to $9 \mathrm{MeV}$. The strategy for the adaptation of contrast is based on the use of X-ray images of step wedges of different materials, which give a unique response curve for a given system. Using these images, we express, for a given thickness of material, the attenuation value of one system as a function of another. The equation of this curve corresponds then to the contrast conversion function, which is applied after the geometric conversion.

Finally, several noise reduction algorithms were evaluated in order to select the best one, $B M 3 D[10]$ to reduce the noise to the level of the reference scanner, without affecting the image quality (e.g. the texture or the sharpness).

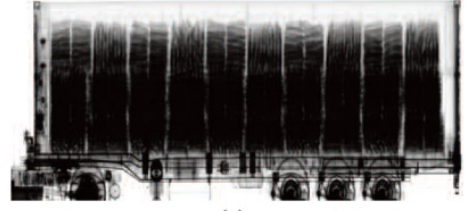

(a)

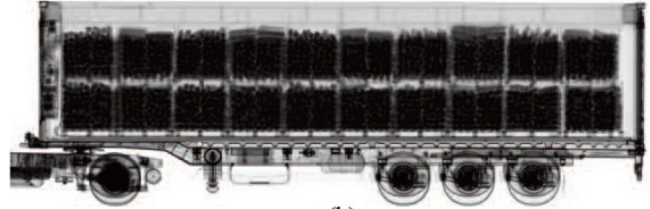

(b)

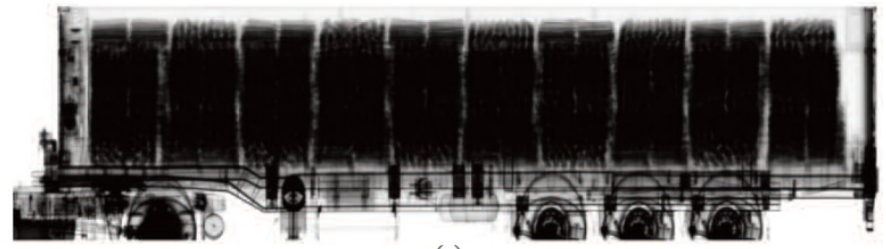

(c)

Figure 10 Real container scan with Euromax (a) and SH-HCVS (b) scanners. The Euromax scan was converted to the HCVS format (c).

\section{Customs Officers Training}


information into consideration, or to ignore it when they believe that the ATR function has generated a false alarm. The waybill information is also provided to the officer, regarding the container contents.

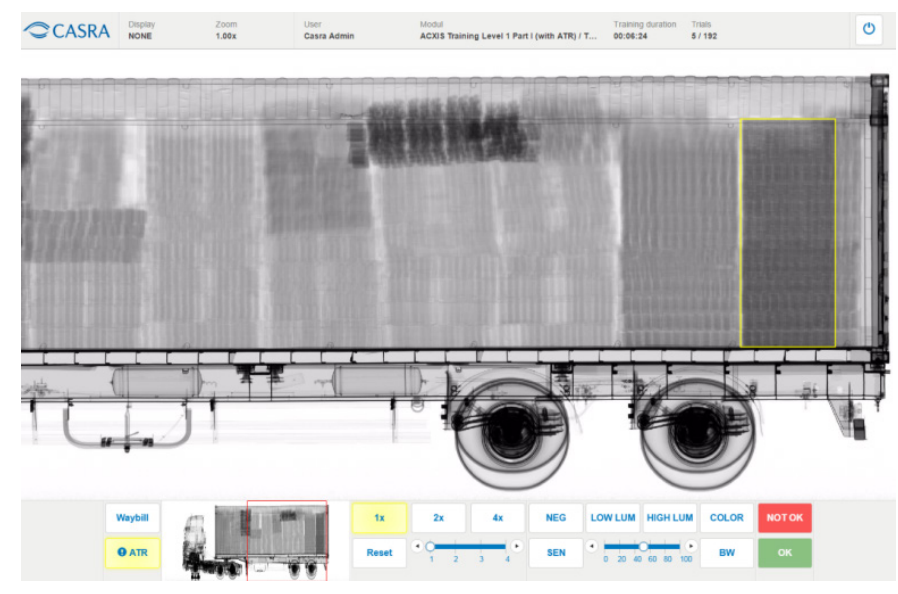

The CBT software incorporates both training and testing modes. The training mode can be used for initial and recurrent $\mathrm{X}$ ray image interpretation training using the large reference database. For each container image, the CBT asks the screening officer whether and where suspect goods are present. Following each image inspection, the officer is given feedback. When a screener correctly reports that an X-ray image contains an item that does not match the waybill or is illicit, the response is counted as a hit. If an incorrect illicit item present answer has been given, the response is categorised as a false alarm. Feedback on missing illicit items and correctly reporting that a container image is harmless is also provided. When an illicit item is present in the image, the feedback includes an additional window displaying the illicit item as a photograph and as an X-ray image. The training time and further details of the simulated scenario can also be examined. Upon completion of a training session, a summary feedback on detection performance in that session is provided. The testing mode is similar to the training mode but feedback is not provided. It can be used to measure X-ray image interpretation competency of customs officers and to conduct research for example on training progress and the value of ATRs.

\section{ATR Algorithms Trained on Merged Threats}

In comparison with the amount of data required to train and test ATR algorithms, a small sample of images containing illicit goods is available. Building a database derived from merging techniques is therefore crucial, especially for machine learning based algorithms where hundreds of thousands of images are often needed. Thus, a single sample can easily be projected according to different orientations and scales, increasing the variability of the dataset.

Our experience has shown that some differences may appear between the results yielded on artificially generated and real testing sets. Though, while tested on real data, ATR algorithms indicate highly satisfactory performances.

In the framework of the ACXIS project, two prominent ATR tools have been developed and trained on projected images: the Automatic Firearm Detection (Figure 12 (a)) and Reefer Unit Analysis (Figure 12 (b)) algorithms.
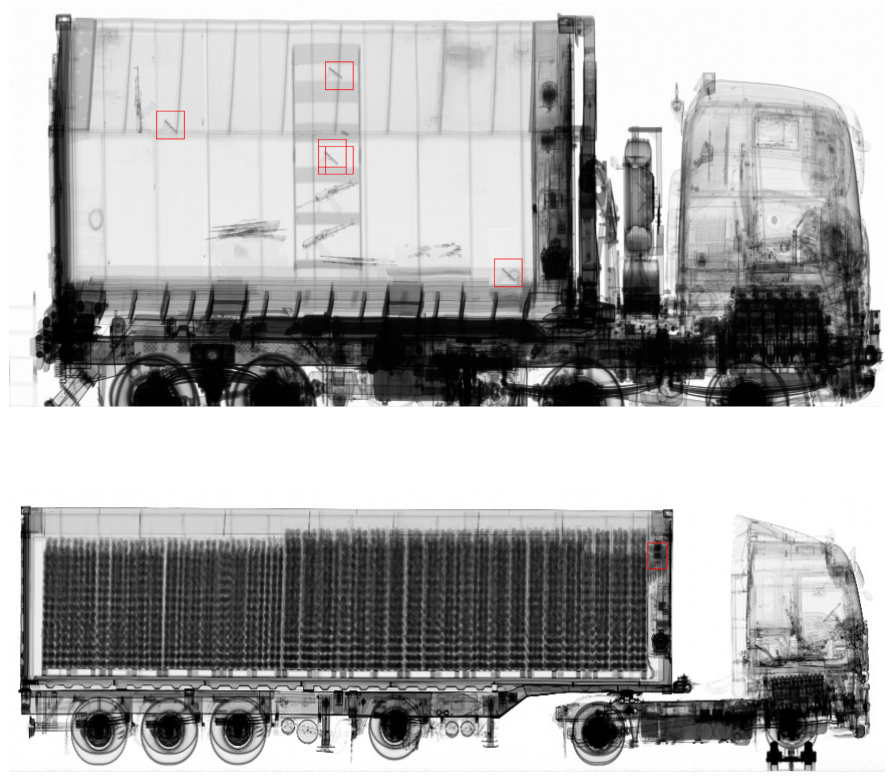

Figure 12 (a) Firearms automatically identified in the container scanned by an HCVM system (b) Narcotics automatically detected in the reefer unit, Rotterdam HCVS, 2016.

\section{Conclusions}

This work, which was part of the ACXIS project, developed a manufacturer independent reference database for X-ray images of illicit and non-illicit cargo, procedures and algorithms to uniform X-ray images of different cargo scanners and measurement parameters, and an automated identification method of potentially illicit cargo. Historic images of real detection scans, images of illicit cargo mock-ups as well as images of non-illicit cargo were integrated into the reference database. The resulting database was then used for the improved training and evaluation of customs' officers. The developed software incorporates ATR functions, which together with the impact of the systematic computer-based training is expected to confirm the benefits through a validation study. The foreseen results of the study will confirm that this software increases the success of border controls and reduces the required time necessary for cargo inspection.

\section{Conflict of Interest}

The authors declare no conflict of interest.

\section{Acknowledgment}

Project ACXIS is supported by the European Commission through the Seventh Framework Programme (FP7). 


\section{References}

[1] S. Michel, M. Mendes, J. C. de Ruiter, G. C. Koomen, and A. Schwaninger, "Increasing X-ray image interpretation competency of cargo security screeners," Int. J. Ind. Ergon., vol. 44, no. 4, pp. 551-560, 2014.

[2] A. Bolfing and A. Schwaninger, "Measurement formulae for image-based factors in X-ray imagery," VICOREG Technical Report, 2007.

[3] A. Bolfing, T. Halbherr, and A. Schwaninger, How image based factors and human factors contribute to threat detection performance in X-ray aviation security screening. Springer, 2008.

[4] S. Michel, S. M. Koller, J. C. De Ruiter, R. Moerland, M. Hogervorst, and A. Schwaninger, "Computer-based training increases efficiency in X-ray image interpretation by aviation security screeners," in Security Technology, 2007 41st Annual IEEE International Carnahan Conference on, 2007, pp. 201-206.

[5] F. Sukowski, T. Fuchs, and P.-M. Keßling, "High energy X-ray CT of freight containers," in Fraunhofer Symposium Future Security. 4th Security Research Conference 2009, 2009, pp. 277-281.

[6] G. Chen, P. Bjorkholm, T. R. Fox, Z. Wilson, and X. Bonsergent, "X-Ray Cargo Inspection: Status and Trends," in Applications of Accelerators in Research and Industry: Twentieth International Conference, 2009, vol. 1099, pp. 570-573.

[7] E. A. Miller, J. A. Caggiano, R. C. Runkle, T. A. White, and A. M. Bevill, "Scatter in cargo radiography," Appl. Radiat. Isot., vol. 69, no. 3, pp. 594 603, 2011.

[8] N. Reims, T. Schoen, M. Boehnel, F. Sukowski, and M. Firsching, "Strategies for efficient scanning and reconstruction methods on very large objects with high-energy x-ray computed tomography," in SPIE Optical Engineering \& Applications, 2014, pp. 921209-921209.

[9] A. Mackenzie, D. Dance, A. Workman, M. Yip, K. Wells and K. Young, "Conversion of mammographic images to appear with the noise and sharpness characteristics of a different detector and x-ray system," Medical Physics, vol. 39, no. 2721, 2012.

[10] K. Dabov, A. Foi, V. Katkovnik and K. Egiazarian, "Image denoising by sparse 3D transform-domain collaborative ltering," IEEE Transactions on Image Processing, vol. 16, no. 8, 2007.

[11] S. M. Koller, D. Hardmeier, S. Michel and A. Schwaninger, "Investigating training, transfer and viewpoint effects resulting from recurrent CBT of X-Ray image interpretation," Journal of Transportation Security, vol. 1, no. 2, pp. 81-106, 2007.

[12] T. Halbherr, A. Schwaninger, G. R. Budgell and A. Wales, "Airport Security Screener Competency: A Cross-Sectional and Longitudinal Analysis," International Journal of Aviation Psychology, vol. 23, no. 2, pp. 113-129, 2013. 